\title{
Phytochemicals for Bacterial Resistance - Strengths, Weaknesses and Opportunities
}

Author

Affiliation

\author{
Simon Gibbons
}

Centre for Pharmacognosy and Phytotherapy, The School of Pharmacy, University of London, London, U.K.

\author{
Key words \\ - Plant-antibacterials \\ - MRSA \\ - Mycobacterium \\ - multidrug-resistance \\ - efflux pump inhibitors \\ - bioprospecting \\ - biowarfare
}

received January 24, 2008 revised March 12, 2008 accepted March 16, 2008

\section{Bibliography}

Dol $10.1055 / \mathrm{s}-2008-1074518$ Planta Med 2008; 74: 594-602 (c) Georg Thieme Verlag KG Stuttgart · New York Published online April 29, 2008 ISSN 0032-0943

\section{Correspondence}

\section{Simon Gibbons}

Centre for Pharmacognosy and Phytotherapy

The School of Pharmacy University of London 29-39 Brunswick Square London WC1N $1 \mathrm{AX}$

U.K.

Tel.: +44-207-753-5913

Fax: +44-207-753-5909

simon.gibbons@pharmacy.ac.uk

\section{Abstract}

This review covers some of the opportunities which currently exist to exploit plants for their natural products as templates for new antibacterial substances. This is a timely exercise given the continuing and developing problems of bacterial resistance, and in particular multidrug-resistance (MDR). Some of the challenges which are evident with bacterial resistance will be described and the strengths and weaknesses of plant natural products are highlighted. Opportunities to characterise antibacterial compounds from several key taxa are described with activity against methicillin-resistant Staphylococcus aureus (MRSA), MDR variants of this species and Mycobacterium tuberculosis (MTB). These pathogens continue to cause problems in terms of their eradication and spread and MTB strains which are extremelydrug resistant (XDR) promise to afford an additional challenge for clinicians. The review also covers plant natural products that modulate or modify bacterial resistance. Specific examples include plant-derived efflux pump inhibitors (EPIs) which inhibit bacterial antibiotic efflux mechanisms that are problematic due to their broadness in substrate specificity. A summary on future trends and directions in this fruitful and interesting area is also given.

\section{Introduction \\ $\nabla$}

One of the most widely-known species of bacteria responsible for many hospital acquired infections is methicillin-resistant Staphylococcus aureus (MRSA) which emerged in the early 1960 s only one year after methicillin was introduced [1]. This species is a Gram-positive 'grape' shaped or coccus like bacterium which derives its specific epithet of aureus due to the golden colour of colonies grown on solid media. This bacterium is a highly 'talented' organism capable of causing skin infections and food poisoning through to clinical problems such as medical device colonisation. It is commonly responsible for wound-related infections and most worryingly for life-threatening conditions such as bacteraemia, necrotising pneumonia and endocarditis [2], [3]. This species is characterised by acquisition of $\beta$-lactam resistance, in particular resistance to methicillin and oxacillin via expression of altered penicillin-binding protein 2' [4]. MRSA strains also produce $\beta$-lactamase enzymes which are responsible for the degradation of these antibiotics [5]. S.aureus is a commensal organism with the ability to thrive on skin and hair and has a high tolerance to salt. The bacterium also has exceptional ability to acquire resistance with many examples of resistance to the tetracyclines, macrolides, aminoglycosides and fluoroquinolones. It is, however, the ability of this bacterium to become resistant to the antibiotics of choice that are used in its treatment which is a major source of concern, specifically to the glycopeptides such as vancomycin which is given intravenously and the first report of intermediate resistance occurred in 1997 [6]. This was followed shortly after by fully vancomycin-resistant strains (minimum inhibitory concentrations greater than $32 \mu \mathrm{g} / \mathrm{mL}$ ) in 2002 [7]. Unfortunately, there have also been occurrences of resistance to some of the newer antibiotics which are used specifically to treat MRSA infections and these include Zyvox (a member of the oxazolidinones) [8] and cubicin (daptomycin a lipopeptide) [9]. Synercid is a mixture of quinupristin/dalfostin (Q/D) and has been approved for treatment of infections caused by MRSA and vancomycin-resistant Enterococcus faecium, however, a recent study has worryingly described strains of $E$. faecium 
which are intermediately-resistant to Q/D without prior use of this agent or of the structurally related veterinary antibiotic virginiamycin [10]. Zyvox was released in 2001 as the first member of the oxazolidinones and is the only new class of antibacterial for 30 years. If we are unable to control the spread and appearance of resistance through appropriate antibiotic use, this inexorable drive of resistance must be met by a continual pipeline of new antibacterial compounds and, more importantly, new templates with novel modes of action.

In the United Kingdom there has been enormous coverage of MRSA and Clostridium difficile infections in the hospital setting and these bacteria have been headline news in the popular media. This is largely a result of the devastating impact these bacteria can have on patients who have undergone surgery, in particular on the old and the young. The UK Office of National Statistics has continually monitored this situation with the regular publication of the statistics detailing the number of death certificates in England and Wales which cite MRSA. The first 2007 figures make disturbing reading and the number of citations has risen dramatically from 2001 (1211) to 2005 (2083) [11]. A number of measures have been put in place in UK hospitals and public health facilities to reduce these occurrences and these include the introduction of antiseptic gels and lotions at the entrance to wards for patients, visitors and healthcare professionals to use on entering and leaving the facility. The government also launched a UK-wide 'Clean Your Hands' campaign aimed at all personnel to highlight the risks associated with the spread of bacteria and to drive the use of antiseptic materials in hospitals by all. This campaign is likely to prove successful but does of course rely heavily on compliance. Staphylococcus aureus has the ability to survive for short periods on floors, walls and furniture where it can be readily picked up and transferred by hands. Improved ward cleaning and maintaining unit cleanliness are also vital to reduce the number of incidences of hospital acquired infection. A return to the 1950's approach of a full cleaning strategy in wards will also reduce this problem with surface sterilisation of floors, furniture and beds on a regular basis. In the UK there has been a move to 'contract out' such procedures to outside companies who may not be as committed or scrupulous in their approach to this need.

MRSA strains may be considered to be a clinical problem but are also responsible for increasing instances of community infections. One global problem that we currently face is that of tuberculosis (TB) which is caused by Mycobacterium tuberculosis (MTB). The statistics associated with this bacterium and infection are far more worrying than those for MRSA. The WHO has published figures which indicate that there is one new infection from MTB every second [12]. This means that during the course of a 45 minute lecture there will be 2700 new infections, or 86,400 infections in one day alone. So by the completion of the conference on medicinal plant research in Graz there will be 432,000 new TB infections! This is truly staggering and easy to see why a third of the world's population ( 2.2 billion people) are infected with the TB bacillus. Current figures indicate that $5-10 \%$ of these ( $110-220$ million) will become sick or infectious, therefore, increasing the likelihood of further infection. Mycobacterium tuberculosis is a filamentous 'acid-fast' bacterium, a common component of soil and a member of the Actinomycetales class of organisms which includes other soil bacteria such as Streptomyces. This bacterium also has the ability to acquire resistance to multiple antibiotics and we have seen the emergence of multi-drug resistant-TB (MDR-TB) which has pos- sibly arisen as a result of inconsistent or partial treatment and this may be as a result of poor patient compliance. This then increases the burden of this disease by the necessary use of second-line anti-TB drugs such as aminoglycosides, polypeptides, fluoroquinolones, ethionamide and $p$-aminosalicylic acid and this has considerable impact on the expense involved and the treatment time which can be as long as two years. Patients with TB who have HIV infection also have increased mortality. Unfortunately strains have arisen which are extremely-drug-resistant, so called XDR-TB [13] and these are described by the Centers for Disease Control in the US as strains resistant to isoniazid and rifampicin (MDR-TB) but also resistant to "any fluoroquinolone and at least 1 of the 3 injectable drugs used in ТВ treatment: capreomycin, kanamycin and amikacin" [14], [15]. This greatly complicates treatment regimens and increases mortality.

However, these statistics and resistance profiles are only certain facets of TB as a condition and we should not lose sight of the debilitating effects of this disease to patients, their families and their community, and it is these very human problems which mark TB as a dreadful disease, but one which is ultimately curable.

\section{The Strengths of Phytochemicals to Treat Bacterial Resistance \\ $\nabla$}

So why should we invest time and effort into characterising new antimicrobial substances from plants? Well, the need to counter bacterial resistance is obvious and pressing. The strengths of phytochemicals in this area are less obvious but certainly compelling. Firstly, in terms of plant chemical ecology, it is logical that plants produce antibacterial metabolites as part of their chemical defence strategy to protect themselves against microbes in their environment and this includes many Gram-positive bacteria. Soil is rich in bacteria, fungi and viruses and it is likely that plants contain latent antimicrobials or synthesise them de novo as part of a phytoalexic response on microbial invasion. Some species of soil bacteria such as Streptomyces are plant pathogens and these are taxonomically related to Mycobacterium species and this opportunity or taxonomic "closeness" and specificity might be exploitable.

Secondly there are countless examples of plants which are used topically and systemically to treat bacterial infections in the ethnobotanical setting [16]. These include Goldenseal (Hydrastis canadensis), Echinacea species and even Umkaloabo which is a traditional South African medicine which has been used to "treat" TB infections and this is based on the roots of Pelargonium reniforme and Pelargonium sidoides. There have to be opportunities to investigate ethnobotanical antibacterials to discover new drugs, particularly given the long association and plethora of herbal materials which are used in this area. Before the advent of antibiotic therapy and even topical synthetic antibacterials, plants were widely accepted as a resource of antiseptic materials, with fresh plants of certain taxa producing an array of volatile natural products with antibacterial activity [17].

Thirdly it is the extensive functional group chemistry, chirality and ultimately chemical diversity of phytochemicals, and natural products in general, which mark them out as a valuable pool of bioactive molecules. $25 \%$ of all prescription medicines owe their origin either directly or indirectly to natural products and in the fields of antibiotics and anticancer drugs this figure is 
nearer to $60 \%$. It is therefore logical to prospect in this area and highly probable that further research will yield new antibacterials. There is considerable research into characterising mammalian cytotoxic (anticancer) natural products, but the possibility arises that extracts and their components may very well exert effects which are specific to bacterial cells.

Finally, phytochemicals are structurally distinct from microbially derived antibiotic natural products such as the tetracyclines and macrolides. It is likely that this chemical uniqueness will give rise to classes of antibacterial which have modes of action which are distinct from existing compounds, e.g., protein synthesis inhibition. Whilst the complete genome of S. aureus has been published, there is much to be done on examining the primary biochemistry of this organism and it might be that phytochemicals display new mechanisms of action, or activity toward some of the newer targets which are becoming more thoroughly investigated such as type II fatty acid synthase (FAS-II) [18].

These four points make a compelling and convincing argument for the evaluation of plants as a source of new antibacterial agents, however we must take a balanced view and look into the reasons why currently we have no plant-derived antibacterials which are used clinically.

\section{The Weaknesses of Phytochemicals to Treat Bacterial Resistance \\ $\nabla$}

The first weakness in this area is one which concerns natural product research in general and bioprospecting in particular. Pharmaceutical companies have largely withdrawn from inhouse natural product research and "farm out" this work to smaller biotech organisations. This means that there is less investment in the area and surprisingly large Pharma is still in the grip of the dogma of combinatorial chemistry. This approach, where tens of thousand of compounds can be synthesised readily and quickly was adopted in the early to mid $1990 \mathrm{~s}$, with the hope of generating significant "in-house" chemical diversity which can be screened by high-throughput screening (HTS) to find leads quickly. Unfortunately and not surprisingly, this approach has not been fruitful with billions of dollars of investment for negligible return. This is largely as a result of the fact that the libraries produced by this approach lack chirality and extensive functional group chemistry, leading to a lack of chemical diversity and consequently biological activity. This would not have occurred with a structured approach to evaluate natural products from any source. However, pharmaceutical companies are starting to realise that nature is a talented chemist and that her products should not be ignored and we are likely to see an expansion of the evaluation of natural products as drug leads in the next 30 years.

The second weakness of this approach is the market area itself. If one looks at the top 200 best selling brand-name drugs in 2006 [19], one can see some remarkable trends. The number one best selling drug was the HMG-CoA reductase inhibitor Lipitor from Pfizer with sales of $\$ 6.58$ billion dollars. Next on the list was Nexium, the enantiomerically pure isomer of omeprazole which is a proton pump inhibitor (PPI) developed by Astra-Zeneca which made $\$ 4.06$ billion. As we move along the list we can see entries in the top 20 drugs for lifestyle-related diseases including heart disease, further proton pump inhibitors for gastric ailments, and several agents which are used to treat depression, which is in itself, depressing. The top 200 drugs contain many examples which are used to treat lifestyle illnesses and this appears to be a trend and the markets for antibacterial drugs are small in comparison to the cardiovascular, immune-inflammation and cancer areas. This will inevitably have a great impact on investment in this area. The most successful antibacterial from the list is Levaquin ( $25^{\text {th }}$, Johnson and Johnson, sales of $\$ 1.41$ billion) and we have to go down $139^{\text {th }}$ to find an MRSA specific agent - Zyvox (linezolid) which made \$200 million in 2006 for Pfizer. One can readily see why investment is made in finding and developing drugs which are used in treating lifestyle diseases rather than in new antibacterials based purely on market return, especially given that the costs for developing a drug in the antibacterial and anti-inflammation areas are comparable.

Unfortunately there are no anti-TB agents in the top 200 best selling brand-name drugs, despite the urgent need and considerable number of patients in this area, almost certainly because the majority of instances arise in the developing world where there are insufficient resources to pay for these expensive drugs. This awful moral dilemma must be resolved. Despite bacterial resistance increasing it is the market that has driven, and will continue to drive research into characterising new antibacterials. However, XDR-TB could be a highly mobile disease and this may "force" research into this area if there are a greater number of cases of infections caused by these strains in the developed world.

\section{Opportunities for New Antibacterials \\ $\nabla$}

So what opportunities exist in terms of chemical entities in this area? In 2004 I wrote a review on antistaphylococcal plant-derived natural products [20] detailing the classes of phytochemical that display in vitro activity with minimum inhibitory concentrations (MIC) of less than $64 \mu \mathrm{g} / \mathrm{mL}$. This was set at this level as the literature abounds with reports of "antibacterial" compounds with MICs of greater than $100 \mu \mathrm{g} / \mathrm{mL}$, which are poorly active and in reality an MIC of less than $10 \mu \mathrm{g} / \mathrm{mL}$ and ideally less than $2 \mu \mathrm{g} / \mathrm{mL}$ is considered as being of interest to Pharma. Whilst many of the examples in the review are only preliminary reports with little further biological activity (e.g., mammalian cytotoxicity screening, spectrum of activity, mechanisms of action), some of the MIC data are outstanding and several of the classes warrant further evaluation, particularly given the simplicity of their structure and potential route to synthesis. Some of these examples are simple and demonstrate the potential to characterise new antibacterials from plants. The first of these is the readily accessible terthiophene 1 [21] which displays activity at an astounding level against S. aureus (MIC $=0.022 \mu \mathrm{g} / \mathrm{mL}$ ) and this activity occurs in the presence of UV light. This would make an excellent topical antiseptic cream that could be formulated for healthcare professionals to use on entering a hospital ward. Metabolite $\mathbf{2}$ is the well-known acylphloroglucinol hyperforin from St John's wort (Hypericum perforatum) which displays very low MICs against MRSA and penicillin-resistant variants ( $\mathrm{MIC}=0.1-1.0 \mu \mathrm{g} / \mathrm{mL}$ ) [22], [23]. The stability and complexity of this molecule could well prove a major issue as this class is known to undergo oxidation and some analogues are susceptible to keto-enol tautomerism and rotameric forms which can complicate their isolation and structure elucidation. However, as we shall see later, there are opportunities in this class.

The geranylated flavanone $\mathbf{3}$ is a simpler and more accessible target displaying very respectable levels of activity ( $\mathrm{MIC}=3.13-$ 
$6.25 \mu \mathrm{g} / \mathrm{mL}$ ) which could be enhanced by simple medicinal chemistry. This type of compound has also been shown to enhance vancomycin in a synergistic fashion and could be of utility as a potentiation molecule. A further geranylated natural product is the xanthone $\mathbf{4}$ which is very potent toward S. aureus strains with MIC values of $0.3-1.25 \mu \mathrm{g} / \mathrm{mL}$. These metabolites are commonly found in the Guttiferae plant family and, as with 1 and 3, they are readily amenable to modification and synthesis and would make interesting leads in terms of simplicity.

We evaluated the roots of the Chinese medicinal plant Angelica dahurica (Apiaceae) known as Bai Zhi [24]. This plant is also claimed to be useful in treating acne and we were therefore prompted to investigate extracts for anti-staphylococcal activity. This is in some ways an example of why one should not conduct bioassay-guided isolation without dereplication as we isolated the well-known polyacetylene falcarindiol (5) which has previously been shown to be antibacterial. Nevertheless, this compound was active against effluxing multidrug-resistant variants of S.aureus with moderate potency against these strains ( $\mathrm{MIC}=8 \mu \mathrm{g} / \mathrm{mL}$ ). These are again relatively simple metabolites that are chemically accessible and we hypothesise that they exert their effects by inhibition of fatty acid biosynthesis, for example, possible inhibition of FAS-II. We recently evaluated dehydrofalcarindiol which differs from falcarindiol by having a terminal exo-methylene group rather than a methyl group and this compound was inactive at $128 \mu \mathrm{g} / \mathrm{mL}$ indicating that the methyl group is a prerequisite for activity, and possible binding to an active site [25]. It is possible that these metabolites are fatty acid mimics and interrupt the biosynthetic machinery required for fatty acid synthesis with the methyl group being an important facet of substrate recognition.

Compound $\mathbf{6}$ is a very interesting antibacterial natural product and was isolated from Evodia rutaecarpa by Dr Michael Adams working at the University of Graz under the direction of Professor Rudolf Bauer and Professor Franz Bucar. This is a 4-quinolone natural product displaying excellent activity against fast-growing species of Mycobacterium ( $\mathrm{MIC}=2 \mu \mathrm{g} / \mathrm{mL}$ against Mycobacterium phlei) [26]. The position of the double bond in the side chain is important for activity, in the case of 6 which is optimal being an 8-9 cis orientation. A synthetic route has been worked out by the Graz team and a patent application has been filed [27].

The final compound from this group is arguably the most intriguing, 7. This simple metabolite was isolated by Thomas Rüegg as part of a team with Professor Gupta and Professor Hostettmann from a pepper species (Piper multiplinervium) in an ethnobotanical study [28]. This compound is a farnesylated salicylic acid derivative with excellent starting potency against the Gram-negative pathogen Pseudomonas aeruginosa. This is an astounding finding as Gram-negative bacteria are generally much harder to find "hits" against, presumably as a result of their outer cell wall which greatly decreases permeability and because they are intrinsically resistant through the expression of membranebound efflux pumps. In the case of Pseudomonas aeruginosa, a number of efflux pumps such as MexABOprM have been described [29] and these confer resistance to multiple antibiotics such as fluoroquinolones and penicillins. The activity of $\mathbf{7}$ is therefore of great interest on a number of levels, least of which is the importance of eradicating $P$. aeruginosa in patients with burns and cystic fibrosis. Compound $\mathbf{7}$ is simple and many analogues could be readily made to dissect activity and even enhance potency. Additionally this metabolite could be formulated as a topical an- tiseptic material and its salicylic acid nature also raises the question of whether this is an anti-inflammatory natural product in addition to being an antibacterial.

Whilst the above compounds are in the majority of cases from preliminary studies, they show the range of phytochemistry available to those interested in new antibacterials and of course display the levels of activity one would expect from a lead compound. These points show that plants are undoubtedly a valuable source of new antibacterial substances and this encouraged our further research in this area.

In particular it was the activity of hyperforin which drove us to study the taxa which produce these molecules. As previously mentioned the activity of this molecule is noteworthy and was first described in 1971 by Gurevich and co-workers [22] and latterly by Schemp et al., who conducted evaluation against MRSA strains [23]. Whilst hyperforin is not an ideal candidate in terms of stability, it is a member of the acylphloroglucinol class of natural product based on the core of $\mathbf{8}$. This is a simple 1,3,5-trihydroxylated benzene (phloroglucinol) possessing an acyl chain which may be simple or complex and multifunctional. Sinaicinone (9) is a member of this class from Hypericum sinaicum [30] and it is not always apparent that these molecules are in fact acylphloroglucinols, particularly given that sinaicinone is in the triketone form of this class and an adamantyl derivative. This metabolite exhibits the best possible features of natural products in general, being highly functional with many pendant prenyl, geranyl and acyl chains and exhibiting a high degree of chirality in the class as a whole.

Other acylphloroglucinols are also antibacterials with some lovely phytochemical examples such as drummondin E (10) with an MIC of $0.39 \mu \mathrm{g} / \mathrm{mL}$ and chinensin I (11) from Hypericum chinense [31] with an MIC of $3 \mu \mathrm{g} / \mathrm{mL}$ again showing that class has potential.

All of these factors prompted us to investigate the genus Hypericum as a source of potential antibacterial leads and we contacted the Royal Botanic Garden Kew at Wakehurst Place who house the UK living national Hypericum collection. From these, through a material transfer agreement, we collected thirty-four species and varieties of Hypericum, and extracts were prepared and screened against an MRSA strain which also expresses the TetK multidrug-efflux pump which confers a high level of resistance to tetracycline. We were somewhat surprised at the high hit rate (32/34) we received for these extracts, particularly that the test strain was an effluxing variant and an MRSA [32]. Profiling of the extracts by TLC and HPLC also indicated that it was not just one active component present (i.e., hyperforin) that was responsible for the bioactivity and the extracts were very different from each other indicating highly varied chemistry. Furthermore the MIC values of some of these extracts were $64 \mu \mathrm{g} / \mathrm{mL}$ and given the high level of complexity of the extracts would indicate the presence of active compounds which possibly exhibit sub $1 \mu \mathrm{g} / \mathrm{mL}$ potency. We therefore embarked on a bioassay-guided isolation approach on several of these species. The first of which was Hypericum foliosum, a species that is endemic to the Azores. Vacuum-liquid chromatography followed by preparative HPLC with monitoring of activity of all fractions resulted in the isolation of $\mathbf{1 2}$ [33]. This is a much simpler and more stable product than hyperforin and is essentially a triprenylated phloroglucinol, with one of prenyl groups being the acyl chain. The other prenyls are the classic 2-methylbutenyl and epoxidised 2-methylbutenyl moieties. This metabolite exhibited moderate activity ( $16 \mu \mathrm{g} / \mathrm{mL})$ and is currently the target of a syn- 


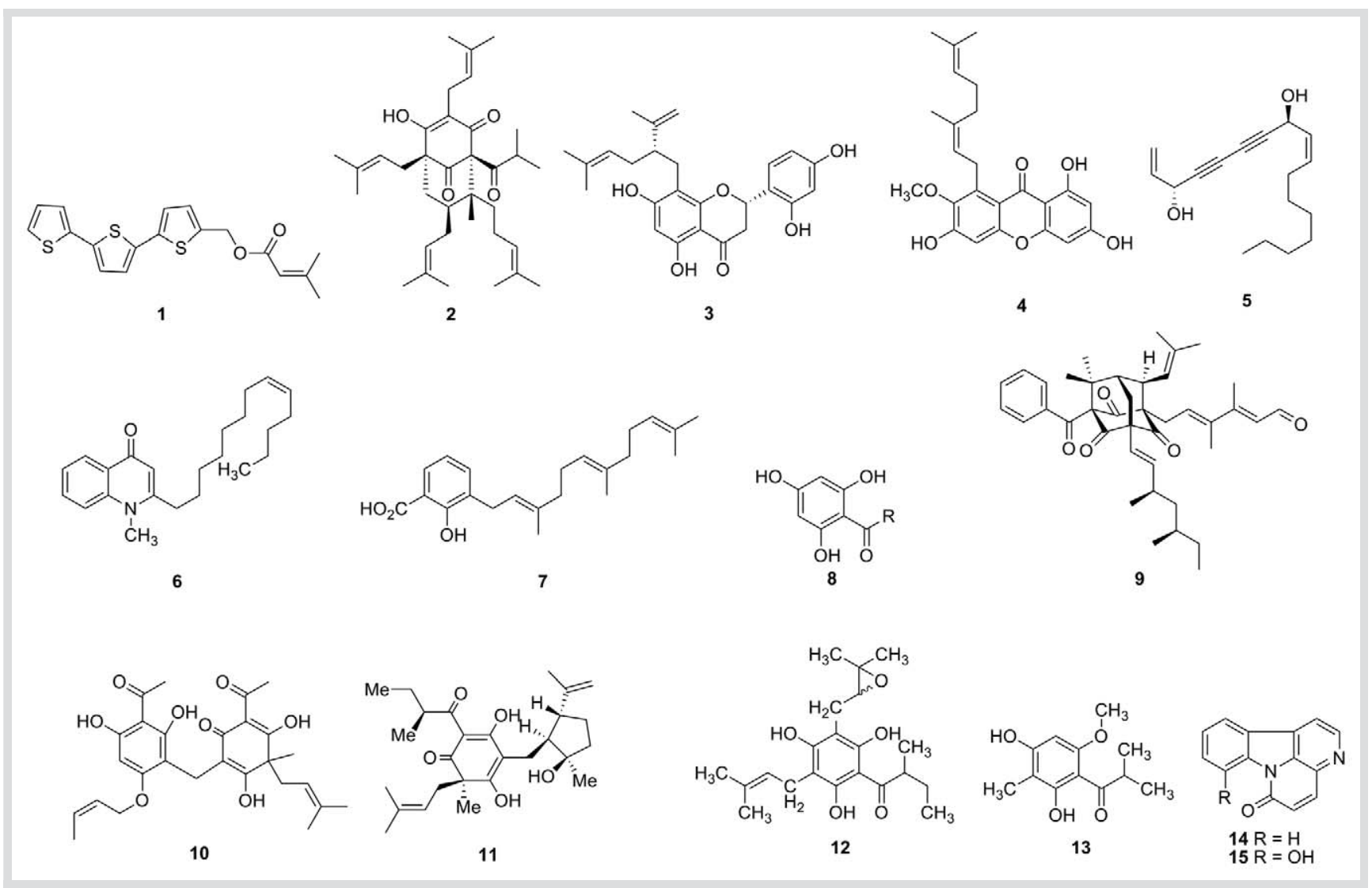

thesis project by the team of Dr Mark Searcey at the University of East Anglia.

The second example from this area was Hypericum beanii which is of Chinese origin and afforded an even simpler molecule (13) with an identical potency [34]. This compound even lacks prenyl groups although a different acyl chain, 2-methylpropanoyl, is present rather than a 2-methylbutanoyl group as seen in 12, and this chain is presumably important for bioactivity.

We have recently filed a patent application on a related metabolite from another species of Hypericum, and the structure of this compound cannot be disclosed at present although the MIC values against a panel of MRSA and MDR strains are in the range of $0.5-1.0 \mu \mathrm{g} / \mathrm{mL}$ and the activity is confined to Gram-positive organisms [35].

Another family which is sometimes overlooked in terms of antibacterial activity is the Alliaceae. Much work has been done on the chemistry and pharmacological evaluation of the common species of Allium, in particular garlic itself, and other common members of this group including leeks (Allium porum), shallots (Allium cepa var aggregatum) and of course onions (Allium сера). Garlic has a long history of usage as an antibacterial and been renowned as 'Russian penicillin' and has even found clinical utility in the United States as a treatment for TB with some success [36]. The Allium genus comprises some 650 species distributed throughout Europe and Asia and there are many herbal medicinal products in current use, mainly in the cardiovascular area, such as Kwai and Cardioace which are standardised on allicin. Given the well-known antibacterial properties of garlic bulbs [37], this leads to the question as to whether the bulbs of other Allium species are rich sources of antibacterials? This is likely given the fact that soil is a complex mixture of filamentous bacteria which includes Mycobacterium and Streptomyces spe- cies, some of which are plant pathogenic [38]. Allium sativum chemistry is sulphur dominant with classic examples such as allicin, ajoene and a range of other sulphides which may be cyclic or linear. However, the chemistry of the family as a whole is diverse with examples of indole alkaloids, highly oxygenated and complex steroidal triterpenes of the furostane and spirostane type and of course the pigments which make this genus such an attractive target for horticulturists due to the presence of red and blue anthocyanindins. Indeed there has been an explosion of horticultural interest in Allium species because of their ability to produce highly coloured blue, red, violet and pink flowers with attractive complex umbel structures. This chemical richness coupled with a likely ecological role for the production of antibacterial substances in the subterranean part of these plants, drove us to evaluate a number of species for antimycobacterial activity. We have been using a Mycobacterium fortuitum model as it can be used in a class II facility, is fast-growing (the assay is complete in 3 days) and shows a similar susceptibility as $M$. tuberculosis in terms of front-line anti-TB drugs, being susceptible to isoniazid, ethambutol and fluoroquinolones [39]. Once an active compound has been isolated, we then screen against other species such as M. abscessus, M. phlei, M. smegmatis, M. aurum and the slow-growing M. tuberculosis. An evaluation of Allium neapolitanum led to the isolation of two canthinone alkaloids, namely canthin-6-one (14) and 8-hydroxy-canthin-6-one (15), which were active against $M$. smegmatis at a level of 8 and $2 \mu \mathrm{g} /$ $\mathrm{mL}$, respectively [40]. Bulbs of other members of the Alliaceae and the Liliales as a whole are certainly worth investigating. A study of Chlorophytum inornatum (Liliaceae) [41] yielded a member of the rare homoisoflavanone class of natural product 16 which displayed moderate activity $(\mathrm{MIC}=16 \mu \mathrm{g} / \mathrm{mL})$ against M. phlei. This class occurs within the Liliaceae and Hyacintha- 
ceae plant families and deserves further evaluation as antibacterials, particularly given their simple structure and difference from conventional flavonoids which display a plethora of antibacterial properties.

\section{Efflux Pump Inhibitors (EPIs) from Plants}

\section{$\nabla$}

Many clinically relevant bacteria express efflux mechanisms of resistance, for example in S. aureus this includes the NorA protein which extrudes certain fluoroquinolones and antiseptics [42] and in Mycobacterium tuberculosis there is the IniA pump which effluxes isoniazid [43].

These efflux pumps generally fall into categories defined by their substrate specificity. Pumps may extrude certain types of substrates such as the QacA system which occurs in S. aureus and is involved in the transport of quaternary ammonium compounds that are antibacterial and are used as topical antiseptics. This has clear implication in terms of hospital disinfection. Some efflux pumps appear to be specific for certain classes of substrate, for example the TetK system which extrudes certain tetracyclines, including tetracycline itself. Finally there are the so-called multidrug resistance (MDR) pumps that appear to have a broader spectrum of substrate specificity and efflux many structurally unrelated antibiotics and antibacterials from the cell resulting in a low and therefore ineffective concentration [44]. This has a very clear implication in terms of a treatment regimen using antibiotics which are substrates for these mechanisms. The proteins which make up these systems are divided in to 5 distinct families primarily based on amino acid sequence homology [45]. These include the major facilitator (MF) superfamily, the resistance-nodulation-division (RND) family, the small multidrug resistance (SMR) family, the ATP binding cassette (ABC) family and the multiple antibiotic and toxin extrusion (MATE) family. The first three families achieve the energy required to extrude a drug out of the cell via the proton motive force ( $\mathrm{pmf}$ ) in a proton-drug antiport system, whereas the MATE family is driven by the exchange of either protons or sodium ions. The ABC family couples drug extrusion with the hydrolysis of ATP [44]. Efflux of drugs from Gram-positive bacteria is mediated by a single cytoplasmic membrane-located transporter of the MF, SMR or ABC families [44]. The efflux mechanism in Gram-negative bacteria is more complex due the presence of an outer membrane. In these species efflux mechanisms form a tripartite protein channel, which requires a protein situated in the periplasm known as the membrane fusion protein (MFP) and an outer membrane efflux protein (OEP) along with the cytoplasmic-located transporter. It is not uncommon for an organism to code for more than one efflux pump, which may either be expressed constitutively or induced in direct response to the presence of a substrate. Pseudomonas aeruginosa and Escherichia coli produce the AcrABTolC system which is a main member of the RND family in these organisms [46]. This pump is involved in the extrusion of certain tetracyclines and fluoroquinolones.

Our approach has been to screen for inhibitors of these processes, using a phytochemical in combination with an antibiotic. There are a number of reasons to pursue this area, notably that the inhibitor restores susceptibility to the antibiotic and an inhibitor-antibiotic combination has been shown to reduce the rate of emergence of antibiotic-resistant variants. This approach of using an inhibitor-antibiotic combination has also been used in the area of $\beta$-lactamase inhibition and there are a number of products currently on the market (e.g., Augmentin; amoxicillin and clavulanate).

A diverse set of compounds have previously been described in the literature to inhibit efflux processes in S. aureus and many of these come from the phytochemical work of Professor Frank Stermitz [47], [48], [49]. Two reviews conducted by the author [20], [50] show that these are extremely varied in terms of their chemical class and shape and include compounds such as reserpine (17) and even simple flavones (e.g., 18) and isoflavones (19). Other diverse structural groups include the porphyrin phaeophorbide A (20) and even complex acylated glycosides from a Geranium species, e.g., 21 [51].

From the same plant berberine (22) and the flavonolignan methoxyhydnocarpin (23) have been isolated and the activity of berberine which is a broadly toxic quaternary alkaloid is potentiated by methoxyhydnocarpin in combination [47]. This gives rise to the interesting possibility that plants produce both antibacterial compounds and compounds which target bacterial efflux mechanisms presumably to inhibit possible resistance to latent plant antibacterials in bacteria in their environment.

Our work in this area began with a synthetic compound (24) which is based on acridone and tetrahydroisoquinoline alkaloids which are plant metabolites. This compound, known as GG918, was in development by GlaxoSmithKline for the inhibition of mammalian P-glycoprotein which is one of the major efflux mechanisms associated with mammalian cancer resistance. It seemed logical to us that as this compound is an inhibitor of mammalian efflux proteins, it was likely that it could also be an inhibitor of certain bacterial transport processes, particularly those associated with MDR and antibiotic efflux. We incorporated $\mathbf{2 4}$ in growth medium at $10 \mu \mathrm{g} / \mathrm{mL}$ and investigated its ability to potentiate the activity of certain antibiotics against resistant strains ( Table 1) which exert their resistance via efflux [52]. As can be seen, 24 caused an eight-fold increase in the activity of norfloxacin against a norfloxacin-resistant strain over-expressing the NorA efflux pump. This strain normally has an MIC of $32 \mu \mathrm{g} / \mathrm{mL}$ but in the presence of 24 the MIC was $4 \mu \mathrm{g} / \mathrm{mL}$. A similar effect was also seen with other effluxing strains including tetracycline and an erythromycin-resistant S. aureus. This compound compared favourably with the well-known EPI reserpine which caused a four-fold reduction in norfloxacin MIC. This prompted us to evaluate $\mathbf{2 4}$ in an ethidium bromide (EtBr) efflux experiment. Ethidium bromide is a substrate for many efflux pumps and fluoresces inside bacterial cells. Its intensity of fluorescence is therefore related to its efflux from bacterial cells and can be used to evaluate compounds which inhibit efflux processes. Fig. 1 shows the percent reduction of efflux (\% increase in fluorescence) plotted against concentration of $\mathbf{2 4}$ and reserpine using SA-1199B, a cell line overexpressing the NorA antibiotic pump. Compound 24 had an $\mathrm{IC}_{50}$ of $2 \mu \mathrm{M}$ which compared favourably with reserpine with an $\mathrm{IC}_{50}$ of $10 \mu \mathrm{M}$. This encouraging result prompted us to evaluate plant natural products, particularly compounds which have similar structural features, for example being large, lipophilic and alkaloidal. Compound 24 and other EPIs also appear to have the ability to dissociate or become protonated in solution.

One such compound that fits these criteria is the metabolite ergotamine (25), one of the alkaloids from ergot (Claviceps purpurea). This compound displayed no direct antibacterial activity, but in combination with norfloxacin at $20 \mu \mathrm{g} / \mathrm{mL}$, caused a 4-fold reduction in norfloxacin MIC against a resistant strain. In an EtBr efflux experiment, ergotamine had a similar level of 


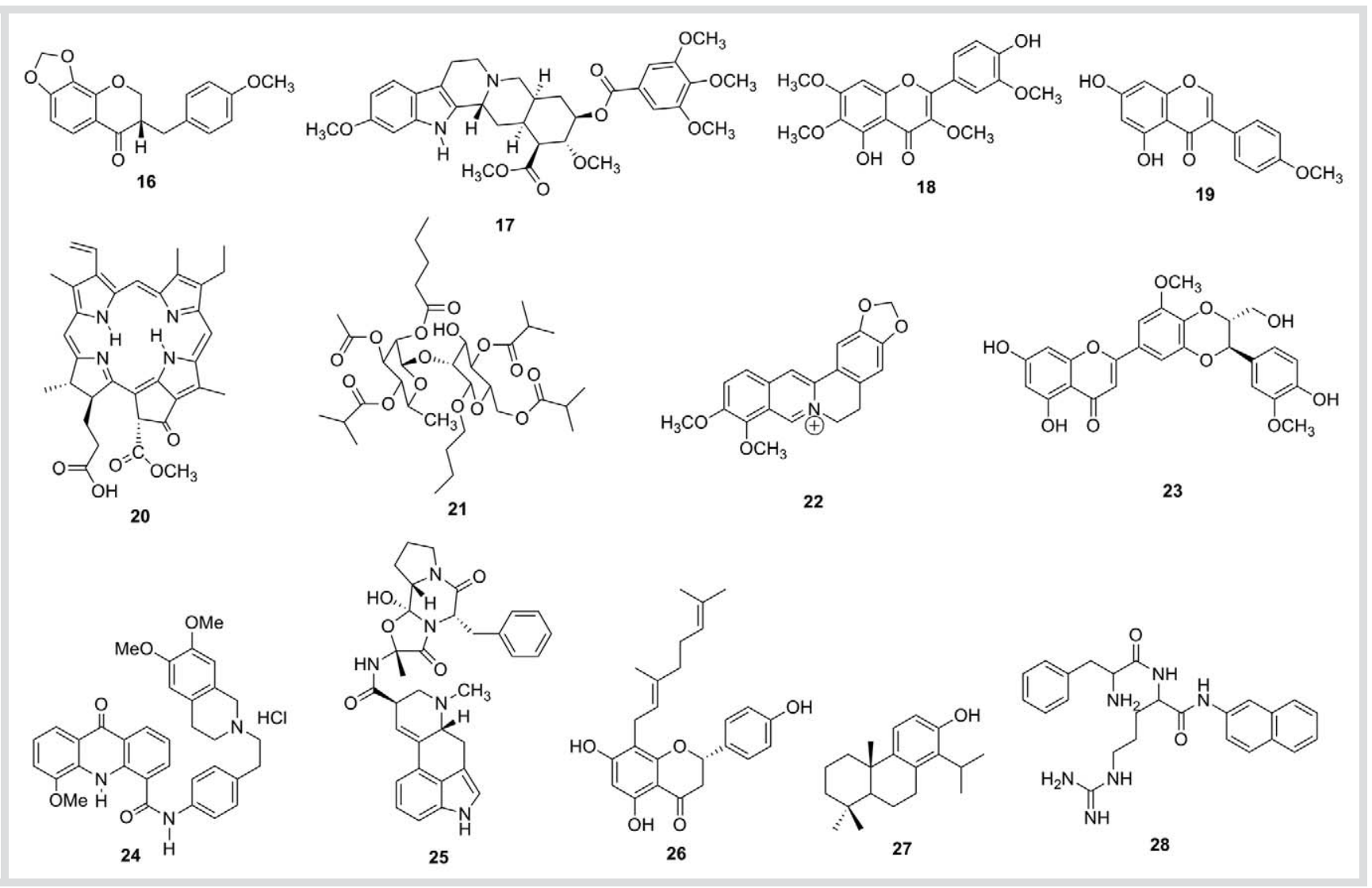

Table 1 Potentiation of norfloxacin by reserpine $(20 \mu \mathrm{g} / \mathrm{mL})$ and $24(10 \mu \mathrm{g} / \mathrm{mL})$ against effluxing and standard strains. Efflux mechanisms are given beneath S. aureus strains in parentheses. Values are minimum inhibitory concentrations (MIC) in $\mu \mathrm{g} / \mathrm{mL}$ and the fold-potentiation is given in bold in parentheses

$\begin{array}{llllll}\text { Antimicrobial } & \text { RN4220 (MsrA) } & \text { CD1281 (TetK) } & \text { SA-1199B (NorA) } & \text { SA-K2068 (MDR) } & \text { ATCC25923 (standard) } \\ \text { Norfloxacin } & 1 & 1 & 32 & 8 & 0.5 \\ + \text { Reserpine } & 0.25(4) & 0.25(4) & 8(4) & 1(8) & 0.125(4) \\ +\mathbf{2 4} & 0.25(4) & 0.25(4) & 4(8) & 2(4) & 0.25(2)\end{array}$

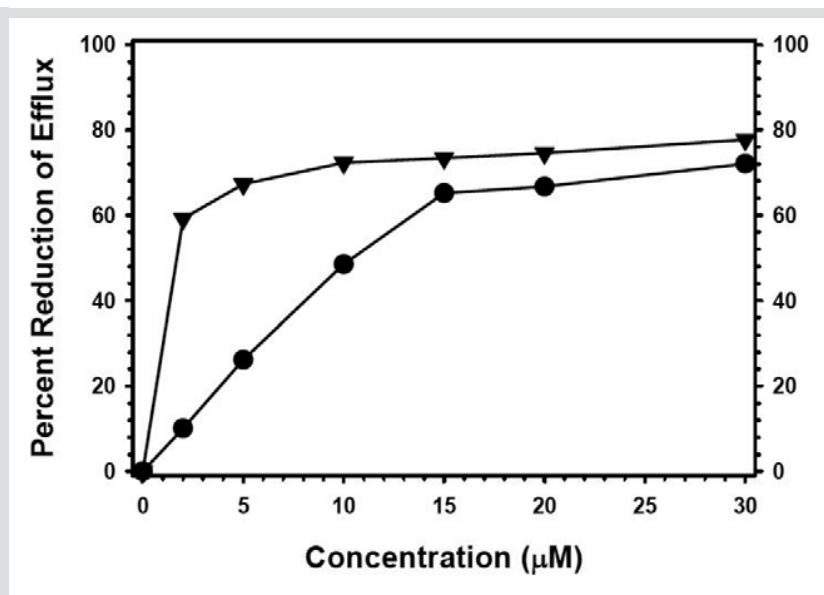

Fig. 1 Ethidium bromide efflux assay for $\mathbf{2 4}$. Effect of $\mathbf{2 4}$ on ethidium efflux; the data presented are means of duplicate experiments $\boldsymbol{\nabla}, \mathbf{2 4} ; \bullet$ reserpine.

potency to reserpine with an $\mathrm{IC}_{50}$ of $7 \mu \mathrm{M}$ [53]. Despite possessing a complex amino acid side chain, ergotamine is very amenable to synthetic modification and a series of analogues could be readily prepared to optimise activity by varying the amino acids at the lysergic acid functionality.

In collaboration with Professor Giovanni Appendino of the University of Novara we have been evaluating the antibacterial activity of some prenylated flavanones and Professor Appendino has been studying these compounds, particularly 8-prenylnaringenin for their interesting ability to inhibit estrogen receptor- $\alpha$ mediated cell growth [54]. A related compound the 8-geranylnaringenin analogue $\mathbf{2 6}$, displayed good antibacterial activity at a level of $2 \mu \mathrm{g} / \mathrm{mL}$ against a panel of wild-type and resistant strains of S. aureus [55]. This compound is large and relatively lipophilic and would have the ability to dissociate in solution. These qualities would make $\mathbf{2 6}$ a good EPI in addition to being an antibacterial substance. Professor Glenn W. Kaatz from the Wayne State University evaluated this metabolite for us in an ethidium bromide efflux assay and $\mathbf{2 6}$ does indeed inhibit the efflux of this substrate and is one of the better EPIs we have evaluated $\left(\mathrm{IC}_{50}=1.5 \mu \mathrm{M}\right)$ [55]. This poses an interesting dilemma. As 26 is antibacterial, could it be exerting its efflux inhibitory effects by disrupting cell metabolism, reducing growth or by causing membrane damage? These phenomena would of course also destroy efflux capability. This leads to the problem of how to devolve antibacterial activity from efflux inhibitory activity? The 
solution for this problem came from Professor Kaatz and his team. One of our group, Dr Eileen Smith, was working on certain members of the Cupressaceae which are rich in antibacterial diterpenes [56]. She isolated the antibacterial compound totarol (27) from Chamaecyparis nootkatensis which has been previously studied extensively by Dr Gary Evans and co-workers as an antibacterial and a potentiator of methicillin against methicillinresistant strains of S.aureus [57]. This compound has also recently been shown to be an inhibitor of bacterial cytokinesis and has low mammalian cell cytotoxicity [58], [59]. We were able to show that at half the MIC of totarol, the MIC of norfloxacin could be lowered 4 -fold against a norfloxacin resistant strain of $S$. aureus. This led to the possibility of totarol also being an EPI. Professor Kaatz and his team were able to investigate this possibility by creating a totarol-resistant mutant lacking the NorA efflux pump and then reintroducing the NorA pump into the mutant by phage transduction. This resulted in a dramatic increase in ethidium bromide MIC and interestingly the MIC of totarol was not affected by the introduction of the NorA pump indicating that totarol is not a substrate for this mechanism. Efflux inhibition studies were conducted and totarol is indeed an EPI with an $\mathrm{IC}_{50}$ of $15 \mu \mathrm{M}$ and this is the first time this dual activity has been resolved for a plant antibacterial [60].

\section{Future Directions for Plant Antibacterials \\ $\nabla$}

The EPIs described here are active against certain S.aureus strains but there is a considerable need to characterise new compounds with activity against other Gram-positive bacteria, for example filamentous bacteria such as Mycobacterium. Current work in this area includes the use of certain flavonoids as bacterial resistance modifying agents with activity against fast-growing mycobacteria [61]. It is, however, the Gram-negative bacteria which promise to be a real future challenge in terms of resistance and these include Pseudomonas aeruginosa, Escherichia coli, Acinetobacter baumannii and Salmonella sp. These bacteria can be intrinsically resistant to a variety of antibacterial substances and at the present time there are very few compounds which have been characterised that inhibit their multidrug efflux mechanisms apart from phenylalanine-arginine- $\beta$-naphthylamide (PABN) (28), which is an inhibitor of the RND pumps MexABOprM and AcrABTolC that are present in P. aeruginosa and Salmonella enterica, respectively. Devolving the pharmacophore of this metabolite has not been achieved to date, although it is likely that a simple structural motif in this molecule is responsible for its ability to bind and inhibit some of these efflux proteins. We are currently investigating plant extracts which have efflux inhibitory activity against strains of S.Typhimurium with Professor Laura J. V. Piddock at the University of Birmingham and hope to characterise some new motifs against these targets [62].

Plant-derived antibacterials also have potential as topical materials rather than systemic drugs and there is currently a need for new classes of compounds to replace old agents such as mupirocin and fusidic acid, which are used topically and to which resistance has occurred in some instances. These new plant-derived topical agents could be used in antibacterial gels, soaps and scrubs and would find utility in hospital wards to contain and reduce the spread of infection. There are considerable benefits to the topical route over the conventional systemic drug, in particular the speed to market and smaller amount of data needed to achieve this. This has been achieved by retapamulin, a fungal- derived antibacterial from Pleurotus mutilus and a member of the pleuromutilin class [63] which has recently been approved by the US FDA for the topical treatment of impetigo. Whilst this natural product is a not a phytochemical, there is no reason why similar usage cannot be found for the plethora of antibacterial plant compounds which exist.

Plant antibacterials could also readily find utility in "medical device patents" as stabilisers and antibacterials. Additionally, there is a need for topical sterilising agents to replace compounds such as triclosan which are incorporated into topical materials and even certain household goods, and plant antibacterials could afford this opportunity.

Plant antibacterials also offer potentially new classes of agent to deal with the threat of biowarfare. A number of bacteria and their toxins have the potential to be used as biowarfare agents and the main risks are from Bacillus anthracis (anthrax), Pasteurella pestis (plague), Franciscella tularensis (tularaemia) and Burkholderia $\mathrm{sp}$. (glanders). The threat from these agents is considerable and the consequences of their use have been demonstrated in a spectacular way by two anthrax attacks in the United States in the past seven years. At the present time, the bacteria listed above are treated by conventional antibiotics such as ciprofloxacin for Bacillus anthracis. What is of considerable concern for governments and health protection agencies is that biowarfare agents could be released which have been engineered to be resistant (for example, by MDR) to all of the commonly used antibiotics. This threat is being taken seriously as the US NIH currently funds a programme at the UK Health Protection Agency's laboratory at Porton Down to screen new antibiotics and new classes of antibacterials for activity against biowarfare agents.

I hope that this review has made a compelling case for plant-derived antibacterials and efflux pump inhibitors. Bacteria are incredibly adept at acquiring resistance and we need a continuing stream of new compounds with new mechanisms of action to circumvent this resistance. This is a vibrant area of research and promises to be more so in the future and I believe that this is an excellent area for young natural product scientists.

\section{References}

1 Jevons MP. 'Celbenin'-resistant staphylococci. Brit Med J 1961; 1: 124 5

2 Yee-Guardino S, Kumar D, Abughali N, Tuohy M, Hall GS, Kumar ML. Recognition and treatment of neonatal community-associated MRSA pneumonia and bacteremia. Pediatr Pulmonol 2008; 43: 203 -5

3 Millar BC, Prendergast BD, Moore JE. Community-associated MRSA (CA-MRSA): an emerging pathogen in infective endocarditis. J Antimicrob Chemother 2008; 61: 1 -7

$4 O^{\prime}$ Hara DM, Reynolds PE. Antibody used to identify penicillin-binding protein 2' in methicillin-resistant strains of Staphylococcus aureus (MRSA). FEBS Lett 1987; 212: $237-41$

5 Moult J, Sawyer L, Herzberg O, Jones CL, Coulson AF, Green DW et al. The crystal structure of beta-lactamase from Staphylococcus aureus at $0.5 \mathrm{~nm}$ resolution. Biochem J 1985; 225: 167 - 76

6 Hiramatsu K, Aritaka N, Hanaki H, Kawasaki S, Hosoda Y, Hori S et al. Dissemination in Japanese hospitals of strains of Staphylococcus aureus heterogeneously resistant to vancomycin. Lancet 1997; 350: $1670-3$

7 CDC. Staphylococcus aureus resistant to vancomycin - United States. Morbid Mortal Weekly Rep 2002; 51: 565-7

8 Hentschke M, Saager B, Horstkotte MA, Scherpe S, Wolters M, Kabisch $H$ et al. Emergence of linezolid resistance in a methicillin resistant Staphylococcus aureus strain. Infection 2008; 36: $85-7$

9 Bennett JW, Murray CK, Holmes RL, Patterson JE, Jorgensen JH. Diminished vancomycin and daptomycin susceptibility during prolonged bacteremia with methicillin-resistant Staphylococcus aureus. Diagn Microbiol Infect Dis; 2007, in press 
10 Karanika M, Prati A, Kiritsi M, Spiliopoulou I, Neonakis I, Anifantaki M et al. Reduced susceptibility to quinupristin/dalfopristin in Enterococcus faecium in Greece without prior exposure to the agent. Int J Antimicrob Agents 2008; 31: $55-7$

11 Office for National Statistics. Deaths involving MRSA and Clostridium difficile continue to rise. Health Statistics Quarterly; 22 February 2007: $1-3$

12 WHO Fact Sheet No 1042007 (http://www.who.int/mediacentre/factsheets/fs104/en/)

13 Goldman RC, Plumley KV, Laughon BE. The evolution of extensively drug resistant tuberculosis (XDR-TB): history, status and issues for global control. Infect Disord Drug Targets 2007; 7: 73-91

14 WHO. Extensively drug-resistant tuberculosis (XDR.TB): recommendations for prevention and control. Weekly Epidemiol Record 2006; 81: $430-2$

15 The tuberculosis X factor. Lancet Infect Dis; 2006; 6: 679

16 Magassouba FB, Diallo A, Kouyaté M, Mara F, Mara O, Bangoura $O$ et al. Ethnobotanical survey and antibacterial activity of some plants used in Guinean traditional medicine. J Ethnopharmacol 2007; 114: 44-53

17 Dupont S, Caffin N, Bhandari B, Dykes GA. In vitro antibacterial activity of Australian native herb extracts against food-related bacteria. Food Control 2006; 17: 929-32

18 Tasdemir D, Topaloglu B, Perozzo R, Brun R, O'Neill R, Carballeira NM et $a l$. Marine natural products from the Turkish sponge Agelas oroides that inhibit the enoyl reductases from Plasmodium falciparum, Mycobacterium tuberculosis and Escherichia coli. Bioorg Med Chem 2007; 15: 6834-45

19 Available at http://www.chem.cornell.edu/jn96/outreach.html. Accessed 2007

20 Gibbons S. Anti-staphylococcal plant natural products. Nat Prod Rep 2004; $21: 263-77$

21 Ciofalo M, Petruso S, Schillaci D. Quantitative assay of photoinduced antibiotic activities of naturally-occurring 2,2':5',2-terthiophenes. Planta Med 1996; 62: 374-5

22 Gurevich AI, Dobrynin VN, Kolosov MN, Popravko SA, Riabova ID. Antibiotic hyperforin from Hypericum perforatum L. Antibiotiki 1971; 16: $510-3$

23 Schempp CM, Pelz K, Wittmer A, Schöpf E, Simon JC. Antibacterial activity of hyperforin from St John's wort, against multiresistant Staphylococcus aureus and gram-positive bacteria. Lancet 1999; 353 : 2129

24 Lechner D, Stavri M, Oluwatuyi M, Pereda-Miranda R, Gibbons S. The anti-staphylococcal activity of Angelica dahurica (Bai Zhi). Phytochemistry 2004; 65: 331 - 5

25 Stavri M, Ford CH, Bucar F, Streit B, Hall ML, Williamson RT et al. Bioactive constituents of Artemisia monosperma. Phytochemistry 2005; 66: $233-9$

26 Adams M, Wube AA, Bucar F, Bauer R, Kunert O, Haslinger E. Quinolone alkaloids from Evodia rutaecarpa: a potent new group of antimycobacterial compounds. Int J Antimicrob Agents 2005; 26: 262 - 4

27 Adams M, Bauer R, Bucar F, Wube AA. Pharmaceutical preparation for treating mycobacterial infections with 4-quinolines from Evodia rutaecarpa. PCT Int Appl WO 2006 094327; 2006

28 Rüegg T, CalderónAI, Queiroz EF, SolísPN, MarstonA, RivasF et al. 3Farnesyl-2-hydroxybenzoic acid is a new anti-Helicobacter pylori compound from Piper multiplinervium. J Ethnopharmacol 2006; 103: $461-7$

29 Li XZ, Nikaido H, Poole K. Role of mexA-mexB-oprM in antibiotic efflux in Pseudomonas aeruginosa. Antimicrob Agents Chemother 1995; 39: $1948-53$

30 Rezanka T, Sigler K. Sinaicinone, a complex adamantanyl derivative from Hypericum sinaicum. Phytochemistry 2007; 68: 1272 - 6

31 Nagai M, Tada M. Antimicrobial compounds, chinesin I and II from flowers of Hypericum chinense L. Chem Lett 1987; 16: 1337-40

32 Gibbons S, Ohlendorf B, Johnsen I. The genus Hypericum - a valuable resource of anti-Staphylococcal leads. Fitoterapia 2002; 73: 300-4

33 Gibbons S, Moser E, Hausmann S, Stavri M, Smith E, Clennett C. An antistaphylococcal acylphloroglucinol from Hypericum foliosum. Phytochemistry 2005; 66: $1472-5$

34 Shiu WK, Gibbons S. Anti-staphylococcal acylphloroglucinols from Hypericum beanii. Phytochemistry 2006; 67: 2568-72

35 Shiu WKP, Malkinson JP, Gibbons S. New antibacterial substances from Hypericum. UK Patent GB0718023.5; 2007

36 Bolton SG, Null G, Troetel WM. The medical uses of garlic - fact and fiction. J Am Pharm Assoc 1982; 22: 40-3
37 Cavallito CJ, Buck JS, Suter CM. Allicin, the antibacterial principle of Allium sativum. I. Isolation, physical properties and antibacterial action. J Am Chem Soc 1944; 66: 1950 -1

38 Healy FG, Wach M, Krasnoff SB, Gibson DM, Loria R. The txtAB genes of the plant pathogen Streptomyces acidiscabies encode a peptide synthetase required for phytotoxin thaxtomin A production and pathogenicity. Mol Microbiol 2000; 38: 794 - 804

39 Tims KJ, Rathbone DL, Lambert PA, Attkins N, Cann SW, Billington DC. Mycobacterium fortuitum as a screening model for Mycobacterium tuberculosis. J Pharm Pharmacol 2000; 52: 135

40 O'Donnell G, Gibbons S. Antibacterial activity of two canthin-6-one alkaloids from Allium neapolitanum. Phytother Res 2007; 21: 653-7

41 O'Donnell G, Bucar F, Gibbons S. Phytochemistry and antimycobacteria activity of Chlorophytum inornatum. Phytochemistry 2006; 67: 178-82

42 Kaatz GW, Seo SM, Ruble CA. Efflux-mediated fluoroquinolone resistance in Staphylococcus aureus. Antimicrob Agents Chemother 1993; 37: $1086-94$

43 Colangeli R, Helb D, Sridharan S, Sun J, Varma-Basil M, Hazbón MH et al. The Mycobacterium tuberculosis iniA gene is essential for activity of an efflux pump that confers drug tolerance to both isoniazid and ethambutol. Mol Microbiol 2005; 55: 1829-40

44 Marshall NJ, Piddock LJ. Antibacterial efflux systems. Microbiologia 1997; 13: 285 - 300

45 Poole K, Srikumar R. Multidrug efflux in Pseudomonas aeruginosa: components, mechanisms and clinical significance. Curr Top Med Chem 2001; $1: 59-71$

46 Poole K. Efflux mediated antimicrobial resistance. J Antimicrob Chemother 2005; 56: $20-51$

47 Stermitz FR, Lorenz P, Tawara JN, Zenewicz LA, Lewis K. Synergy in a medicinal plant: antimicrobial action of berberine potentiated by $5^{\prime}-$ methoxyhydnocarpin, a multidrug pump inhibitor. Proc Natl Acad Sci U S A 2000; 97: 1433-7

48 Morel C, Stermitz FR, Tegos G, Lewis K. Isoflavones as potentiators of antibacterial activity. J Agric Food Chem 2003; 51: 5677-9

49 Tegos G, Stermitz FR, Lomovskaya O, Lewis K. Multidrug pump inhibitors uncover remarkable activity of plant antimicrobials. Antimicrob Agents Chemother 2002; 46: 3133-41

50 Stavri M, Piddock LJV, Gibbons S. Bacterial efflux pump inhibitors from natural sources. J Antimicrob Chemother 2007; 59: 1247-60

51 Stermitz FR, Cashman KK, Halligan KM, Morel C, Tegos GP, Lewis K. Polyacylated neohesperidosides from Geranium caespitosum: bacterial multidrug resistance pump inhibitors. Bioorg Med Chem Lett 2003; 13: $1915-8$

52 Gibbons S, Oluwatuyi M, Kaatz GW. A novel inhibitor of multidrug efflux pumps in Staphylococcus aureus. J Antimicrob Chemother 2003; 51: $13-7$

53 Gibbons S, Kaatz GW. Unpublished results.

54 Brunelli E, Minassi A, Appendino G, Moro L. 8-Prenylnaringenin, inhibits estrogen receptor- $\alpha$ mediated cell growth and induces apoptosis in MCF-7 breast cancer cells. J Steroid Biochem 2007; 107: 140 - 8

55 Appendino G, Kaatz GW, Gibbons S. Unpublished results.

56 Smith ECJ, Williamson EM, Kaatz GW, Gibbons S. Antibacterials and modulators from immature cones of Chamaecyparis lawsoniana. Phytochemistry 2007; 68: $210-7$

57 Nicolson K, Evans G, O'Toole PW. Potentiation of methicillin activity against methicillin-resistant Staphylococcus aureus by diterpenes. FEMS Microbiol Lett 1999; 179: 233 -9

58 Jaiswal R, Beuria TK, Mohan R, Mahajan SK, Panda S. Totarol inhibits bacterial cytokinesis by perturbing the assembly dynamics of FtsZ. Biochemistry 2007; 46: 4211 - 20

59 Clarkson C, Musonda CC, Chibale K, Campbell WE, Smith P. Synthesis of totarol amino alcohol derivatives and their antiplasmodial activity and cytotoxicity. Bioorg Med Chem 2003; 11: 4417-22

60 Smith ECJ, Kaatz GW, Seo SM, Wareham N, Williamson EM, Gibbons S. The phenolic diterpene totarol inhibits multidrug efflux pump activity in Staphylococcus aureus. Antimicrob Agents Chemother 2007; 51: $4480-3$

61 Lechner D, Gibbons S, Bucar F. Modulation of isoniazid susceptibility by flavonoids in Mycobacterium. Phytochem Lett; 2008, in press

62 Rahman MM, Garvey M, Piddock LJV, Gibbons S. Antibacterial terpenes from the oleo-resin of Commiphora molmol (Engl.). Phytother Res; 2008, in press

63 Jones RN, Fritsche TR, Sader HS, Ross JE. Activity of retapamulin (SB275833), a novel pleuromutilin, against selected resistant Gram positive cocci. Antimicrob Agents Chemother 2006; 50: 2583-6 